\title{
Decoding information in multilayer ecological networks: The keystone species case
}

\author{
Claudia A. Huaylla a,b,*, Marcos E. Nacif ${ }^{\mathrm{a}, \mathrm{b}}$, Carolina Coulin ${ }^{\mathrm{a}, \mathrm{b}}$, Marcelo N. Kuperman ${ }^{\mathrm{c}, \mathrm{d}}$, \\ Lucas A. Garibaldi ${ }^{\mathrm{a}, \mathrm{b}}$ \\ a Universidad Nacional de Río Negro. Instituto de Investigaciones en Recursos Naturales, Agroecología y Desarrollo Rural. San Carlos de Bariloche, Río \\ Negro, Argentina \\ ${ }^{\mathrm{b}}$ Consejo Nacional de Investigaciones Científicas y Técnicas. Instituto de Investigaciones en Recursos Naturales, Agroecología y Desarrollo Rural. San Carlos de \\ Bariloche, Río Negro, Argentina \\ c Centro Atómico Bariloche (CNEA) and CONICET, R8402AGP Bariloche, Argentina \\ ${ }^{\mathrm{d}}$ Instituto Balseiro, Universidad Nacional de Cuyo, R8402AGP Bariloche, Argentina
}

\section{A R T I C L E I N F O}

\section{Keywords:}

Betweenness

Ecosystem

Modularity

Random networks

Restoration

Trofic networks

\begin{abstract}
A B S T R A C T
The construction of a network capturing the topological structure linked to the interactions among species and the analysis of its properties constitutes a clarifying way to understand the functioning of an ecosystem at different scales of analysis. Here, we present a novel systematic procedure to profit from the enhanced information derived from considering its multiple levels and apply it to analyse the presence of keystone species.

The proposed method presents a way to unveil the information stored in a network by comparing it to some randomised modification of itself. The randomising of the original network is done by swapping a controlled number of links while preserving the degree of the nodes. Then, we compare the modularity value of the original network with the randomised counterparts, which gives us a measure of the amount of relevant information stored in the first one. Once we have verified that the modularity value is meaningful, we use it to perform a community analysis and a characterisation of other topological properties in order to identify keystone species.

We applied this method to a pollinator-plant-herbivore trophic network as a case study and we found that (a) the comparison between the modularity of the original and the randomised networks is a suitable tool to detect relevant information; and (b) identifying keystone species yields different results in bipartite networks from the ones obtained in networks of more than two trophic levels. We also analysed the effect of eliminating selected species from the system on the cohesion of the network. The selection of these species was made according to the centralities values, such as degree and betweenness, of the corresponding nodes.

Our findings show that our analysis, mainly based on the measure of modularity is a reliable tool to characterise ecological networks. Additionally, we argue that since degree and betweenness are not always correlated, it is more reliable to measure both in an attempt to detect keystone species. The methodology proposed here to identify keystone species can be applied to other ecological networks currently available in the literature.
\end{abstract}

\section{Introduction}

The analysis of species interactions in ecological systems has become a central topic in modern ecology, and particularly mutualistic and antagonistic systems have been studied for years. Due to their ecological relevance and their importance in the economy, a considerable number of these types of systems have been completely surveyed in field studies, and their topological properties have been the object of theoretical studies in the context of complex network theory (Bascompte et al., 2003). In any ecological system, the species are represented by nodes and their interactions by links. Both elements define the structure of the underlying network. Notably, the

\footnotetext{
* Corresponding author at: Universidad Nacional de Río Negro. Instituto de Investigaciones en Recursos Naturales, Agroecología y Desarrollo Rural. San Carlos de Bariloche, Río Negro, Argentina.

E-mail addresses: cahuaylla@unrn.edu.ar (C.A. Huaylla), mnacif@unrn.edu.ar (M.E. Nacif), carocoulin@gmail.com (C. Coulin), kuperman@cab.cnea.gov.ar (M.N. Kuperman), lgaribaldi@unrn.edu.ar (L.A. Garibaldi).
} 
effect of this structure on the dynamics of this type of system is a fruitful field of research and valuable results for abiotic, trophic and mutualistic networks have been obtained (Bascompte and Jordano, 2014; Burgos et al., 2007; Medan et al., 2007; Memmott et al., 2004; Vázquez and Simberloff, 2002). It has not been that long since ecologists began applying network theory to study different ecosystems (Bascompte, 2007). Mapping the connections between species not only reveals the underlying rules that govern the dynamics of ecological networks, but is also useful to inform practices that may help foster biodiversity and strengthen ecosystems to prevent their collapse. In addition, network theory allows us to understand how the underlying topology gives rise to non-trivial emergent behaviour and how the intertwining of individual interactions leads to a stable and complex macroscopic scenario. Despite the inherent difficulties in recording interactions among all the species in an ecosystem, the results obtained encourage researchers to tackle this important task. Among the most crucial concepts associated with network-based studies are that of generalists and specialists, whose relevance has been better reflected in the architecture of interactions between species.

Some results derived from studies based on networks help to characterise biodiversity and its connection with the robustness of ecosystems and to analyse the role of different species within the networked structure. This complex structure of networks not only reveals that it is unlikely that the loss of a single species will cause the collapse of an ecosystem, but it also helps appreciate that not all species play the same role. Many studies have analysed bipartite networks to show how the species' importance (Martín González et al., 2010; Poisot et al., 2014) is reflected in the topology. Here, we are interested in going beyond the traditional paradigm based on bipartite mutualistic and antagonistic networks, understanding the ecosystem as a more complex structure, capable of being represented at various interacting levels. This constitutes our rationale to study networks of more than two trophic levels and their projections, a procedure that can be replicated on all types of networks.

To link this procedure with relevant ecological questions, in this study, we focus on one of the ecological problems entailed in network analysis, namely the ecosystem restoration, for which it is necessary to make a selection of keystone species. Therefore, though the importance of species selection to maintain the structure of an ecological network is central in ecology, it still lacks a systematic approach (Cottee-Jones and Whittaker, 2012; Mello et al., 2015). Different methods have been proposed for the selection of species (Jordán et al., 1999, 2006; Jordán, 2009; Mello et al., 2015), one of which is complex network analysis. Such network representation of the underlying topology of interactions between species helps shed light on the understanding of how an ecosystem functions.

It is worth noting that, there are several definitions of "keystone species" (Cottee-Jones and Whittaker, 2012) and many authors agree that, within a network, it corresponds to nodes with a high degree or with the most abundant species. In addition, Jordán et al. (2006) presented global and local indices with different properties and discussed which questions the different indices answered best. Moreover, they studied the correlation between the indices of a particular network, some of which can be used to determine keystone species. However, the relevance of the information provided by those indices depends on the focus of the objective being sought. Jordán (2009) has posed a new approach for classifying the interaction of each species in complex networks, which consists of characterising all the species' positional importance. Generally, in the selection of keystone species there tends to be an oversimplification when focusing only on local information of nodes, thereby ignoring the global structure of the network. In this research, keystone species are defined as those that determine the structure of the network and whose elimination produces dramatic changes, such as its fragmentation. Interestingly, within an ecosystem, the architecture of networks of mutualistic and antagonist species represents the stability of its communities. Furthermore, understanding the structure of these networks is central when trying to characterise and predict changes in the ecosystem and in the communities associated with a change in species abundance.

In order to achieve the main goal of our study, we resort to the characterisation of networks built from information derived from the collected data and examine its betweenness and modularity. Primarily, our goal is to unveil the existence of relevant coded information in such networks by contrasting their structures with the structure of randomised versions of themselves. Specifically, we want to understand how this information affects the modularity of a network as later we will analyse the community division of the network by recurring to algorithm based on the modularity value. We hereby present a systematic way to gradually increase the degree of randomness in a network and to show how this process affects the modularity due to the deletion of causal correlations derived from the ecological interactions. For this purpose, we develop an algorithm which renders itself useful in generating random networks. This algorithm, thoroughly described here, can be implemented with any other convenient software. Finally, we propose a detailed protocol to determine keystone species. Specifically, our research aims are (1) to determine if the network presents a topology with relevant features that differentiate it from a random one, and (2) to identify the network keystone species. Moreover, the details regarding the construction of the algorithm, the identification of keystone species, and our case study are presented in Section 2, while our results and interpretation are included in Section 3. In Section 4, we present the discussion and the implications of this work.

\section{Materials and methods}

\subsection{Tunable randomisation of a network}

Throughout this research, we consider that meaningful ecological interactions among species give rise to correlations that establish a clear difference between a real ecological network and a random one. These correlations are referred to as the amount of relevant stored information coded by different quantities called indices or centralities. When the structure of the original network presents relevant information about the system, the measured quantities should differ from the ones adopted by a comparable random network. Consequently, the transition from a network to its randomised version, accomplished by performing sequential and controlled changes in its topology in order adds disorder, destroys the present correlations and any stored information. When the indices are tracked through these changes, their values should move from the original ones to those corresponding to a random network with comparable topologies which, in our case, is associated with sharing the same degree distribution.

Several parameters can be calculated to characterise a network, for example, betweenness, degree, modularity, etc. (Boccaletti et al., 2014; Kivelä et al., 2014), some of which are node centred while others provide global information. From this last group, modularity was chosen because it weighs the existence of correlations, and extracts the residual values associated with a random network. We expect this value to monotonically decrease as the degree of randomness increases. It is worth noting that modularity is a simple measure that quantifies the intensity of the intra-community vs. the inter-community links, and it also helps characterise the community structure. Communities are disjoint sets of nodes which most likely share common properties and/or play similar roles within the graph but that are primarily defined by the topology of the network. Particularly, this measure can be calculated by using different algorithms such as the Newman-Girvan (Girvan and Newman, 2002; Newman, 2004a,b) or the Louvain one (Blondel et al., 2008), which was used in our study. Louvain's algorithm groups the nodes in such a way as to maximise the modularity function, considers several groups of nodes and selects the groups that maximise the function. It is also worth highlighting that the algorithm for disordering a network preserves the degree of each node, i.e., the 
degree distribution remains unaltered, where degree is defined as the number of neighbours of a node and can be calculated in undirected and directed networks (Fornito et al., 2016). This disordering algorithm is a null model. Specifically, the mean degree of an undirected network is the average of all the node degrees. On the other hand, directed networks have two types of degrees: (1) the in-degree, which counts the number of incoming edges and (2) the out-degree, which counts the number of outgoing ones.

Additionally, a network can be represented by using an adjacency matrix where the network to be studied is referred to as the original network. In order to compare the generated random networks with the original one, we plot its modularity as a function of the number of changes implemented. If our original network differs from a random one, we expect the modularity to decrease as the network is randomised by performing random changes on its structure.

Our disordering algorithm: (detailed description)

Step 1: Calculate the degree of each node.

Step 2: Make a defined number of swaps $\Delta$ which yields a new adjacency matrix.

We define swap as follows: choose two nodes and two neighbours of these $\left(A_{i j}=1, A_{i^{\prime} j^{\prime}}=1\right), j$ which must not be neighbour of $i^{\prime}$, and $j^{\prime}$ must not be neighbour of $i\left(A_{i j^{\prime}}=0\right.$, $\left.A_{i^{\prime} j}=0\right)$. As we want to preserve the degree of each node, we swap both their neighbours $\left(A_{i j^{\prime}}=1, A_{i^{\prime} j}=1\right.$ ) (Fig. 1).

Step 3: Calculate the number of different links between the original and the new network. To do this, we can compare the final adjacency matrix with the original one.

Step 4: Calculate the modularity of the new network.

Step 5: Repeat steps 2, 3 and 4, several times to get a new network with an increasing degree of disorder each time. Once the modularity has reached a steady value, discontinue these repetitions.

Following the aforementioned procedure, networks with increasing levels of randomness are obtained where the preservation of the degree distribution is guaranteed. It is worth noting that this method allows us to generate networks with a tunable degree of randomness by swapping pairs of links of the original network without changing the degree distribution. To achieve this, at each step, we pick a pair of randomly chosen links and exchange two of the adjacent nodes of each edge. When the procedure leads to a duplicated connection, we reject the proposed change and move forward with the algorithm. The corresponding algorithm is programmed by using RStudio.

In our case, ten networks for each value of $\Delta$ were constructed and their corresponding modularity values were averaged. Furthermore, the previously mentioned algorithm was applied to a network with more than one level in order to determine if its structure was significantly different from that of its randomised versions. The number of swaps is not defined a priori. We analysed how the measured quantity varies as the disorder increases and we stop when the value stabilises and remains almost constant even as the number of swaps increases. We consider that stability has been reached if between a number of swaps $n$ and its double $2 n$ we observe no changes in the modularity. The original multilayer network was projected, as proposed by Boccaletti et al. (2014) , to identify keystone species using known and available tools. Then, the adjacency matrix was built, which, in this instance, included three types of identified nodes.

\subsection{Characterisation of the network topology}

At this point, since the preservation of the degree distribution is guaranteed, it is no longer necessary to carry on analysing its randomised versions. Furthermore, the fact that the original network and its randomised versions share the same degree distribution is an indication that the degree of each node is not a suitable quantity to fully understand its role or importance within the network. However, the degree distribution is a valuable tool to gain insight into the possibility of verifying whether a sample is biased or incomplete. Generally, ecological networks correspond to a power-law degree distribution. There is evidence that biological networks are both scale-free (Jeong et al., 2000, 2001) and hierarchical (Ravasz et al., 2002). Despite the low number of nodes, here we assume that the degree distribution of our ecological network should not be too different from a powerlaw distribution shape. While a random network does not necessarily share this property of biological networks, the ones resulting from the proposed randomisation process will do, as the original degree distribution is preserved.

Nevertheless, if the degree distribution of the original network is too far from a power-law distribution, there is a high probability that the sample is neither complete nor unbiased. On the other hand, it is important to note that the fact that the degree distribution approaches a power-law distribution does not warrant the accuracy of the sample. Using the degree distribution in these very small networks to analyse their completeness could lead to unreliable results. Consequently, this degree distribution limitation will be taken into account when extracting information from it. In particular, at the moment of identifying keystone species, we will focus on the betweenness centrality measure. Notably, the problem of identifying nodes that play a central structural role is one of the main topics in the traditional analysis of complex networks. Thus, there are many parameters that measure the structural relevance of each node, including node degree, closeness, and betweenness (Boccaletti et al., 2014; Kivelä et al., 2014). Hereby, we will focus on two of them: degree and betweenness. As previously mentioned, while the degree of a node can say something about its relevance, it is still a local measure, and as such, lacks global information about the network.

In this work, we are interested in identifying the specific nodes that are responsible for the network's cohesion, which are henceforth considered keystone species. While still being a centrality measure, betweenness, can provide valuable global information. Fundamentally, it measures the proportion of the shortest paths between all node pairs passing through a given index node and connecting parts of the network (Freeman, 1979). Furthermore, if such nodes of high betweenness are deleted, the network structure could collapse. Since it is not enough to ascertain their key role, the verification of their importance to preserve the network architecture must be carried out. Finally, the number of communities is calculated in order to complement this measure.

The use of degree to identify keystone species does not necessarily yield the same results as the use of the betweenness. While it is rather intuitive to link a high degree node with a keystone species, it should be noted that this assumption is based on a method that gauges a particular node but not the network's structure. Here we are looking for species whose disappearance can lead to a total collapse of the ecosystem. Thus we recur to betweenness since despite being a centrality quantity it is a parameter with global reach. Generally, degree and betweenness are not related but sometimes, in ecological networks, they might be correlated (Dormann, 2011). If the elimination of a high degree node produces a dramatic change in the cohesion of the ecological network, its betweenness must be also high. The reciprocal is not necessarily true.

Algorithm for selecting keystone species:

Step 1: Calculate the betweenness value of each node.

Step 2: Normalise each value from step one by dividing it by the highest betweenness.

Step 3: Sort out the list from the highest to the lowest value.

Step 4: Choose a value of $\alpha<\alpha_{c}$, such that the region of maximum slope is completely covered.

Step 5: Calculate the number of values greater than $\alpha . j=$ number of values greater than $\alpha . \alpha \in \mathbb{R}$. 
A

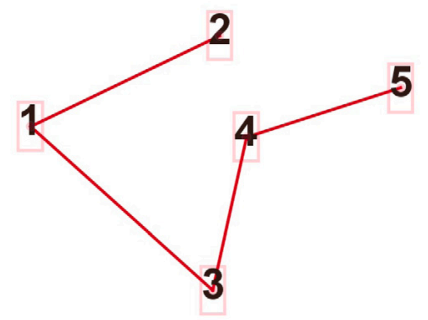

B

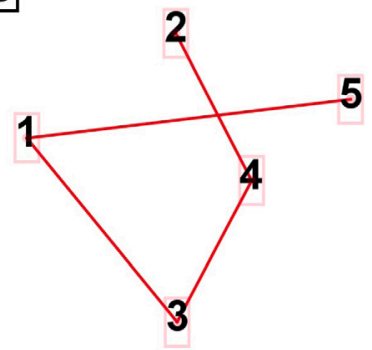

Fig. 1. Simplified representation of the swap procedure. (A) Original graph. $A_{12}=1, A_{45}=1, A_{24}=0, A_{15}=0$. (B) Graph with a single swap. $A_{12}=0, A_{45}=0, A_{24}=1, A_{15}=1$.

Step 6: Delete only node $i$ of the list. Then, calculate the number of communities and connected components.

Step 7: Repeat step five for $i$ in $\{1, \ldots, j\}$.

Step 8: Make a table with the number of communities and connected components before and after deleting each species.

By ranking the values of the normalised betweenness from highest to lowest and plotting betweenness $\left(y_{i}\right)$ vs order $\left(x_{i}\right)$, a curve $f\left(x_{i}\right)=y_{i}$ is obtained. This curve has a point of maximum slope at a certain value $x_{c}$ and we define $\alpha_{c}=f\left(x_{c}\right)$. The points above that value are the ones that will be relevant and that we will call the region of interest. The condition to be fulfilled is $\alpha<\alpha_{c}$.

At this point, we are interested in nodes that when removed, the network begins to lose cohesion, which are henceforth considered keystone species. We choose the nodes whose deletion leads to an increase in the number of communities or connected components into which a network can be divided. Note that the concept of community responds to the use of complex networks and not to the field of ecology. A great number of communities is an indication of a less cohesive network. We will also study which are the keystone species of bipartite networks.

\subsection{Case study. Pollinator-plant-herbivore interactions}

In order to evaluate this protocol, forest data was used to study the relation between plant-pollinator and plant-herbivore interactions. This research was carried out in "El Foyel", located in the Rio Negro province, in Northwestern Patagonia, Argentina. The dataset was collected at the same time and corresponds to that of spring 2014 and summer 2015, and was published as two independent studies, one for plant-pollinator interactions (Coulin et al., 2019) and another one for plant-herbivore interactions (Nacif et al., 2020). For plant-pollinator interactions, we periodically surveyed the number of visitors (potential pollinators) during ten-minute censuses for each flower species (number of visits/flower/ten minutes) throughout the entire flowering season. The number of sampling observations varied across plant species due to differences in flowering duration, abundance, and spatial distribution. Visitors that could not be identified to species level, were recorded either at its family level or grouped as morphotypes. The complete dataset includes 80 visitor species/morphotypes. The percentages are: Order $6 \%$, family $37 \%$, species $37 \%$ and morphotype $20 \%$.

In order to measure arthropod herbivory, twenty-four saplings per species were randomly selected (for more details see Nacif et al., 2020). In each individual, small marked branches at intermediate plant heights were sampled and arthropod herbivory was recorded in 18 leaves per branch. Arthropod herbivory was quantified by computing the frequency of leaf damage (number of damaged leaves/total), following the methodologies proposed by Garibaldi et al. (2011). We classified arthropod herbivores and leaf damage into thirteen different guilds such as leaf chewers, bud feeders, hole feeders, continuous skeletonizers, sticky skeletonizers and patch skeletonizers, surface abrasion feeders (grouped as exophagous feeders), and curvilinear, linear and continuous miners, sap-sucking and wrinkling feeders, and gallers (grouped as endophagous feeders). Protocols and classification were based on McQuillan (2008), Novotny et al. (2010), Garibaldi et al. (2011). Hereafter, we refer to Senescence (Herb1), Leaf chewers (Herb2), Continuous miner (Herb3), Continuous skeletonizers (Herb4), Leaf gallers (Herb5), Hole feeders (Herb6), Linear miners (Herb7), Patch skeletonizers (Herb8), Sap-sucking and wrinkling feeders (Herb9), Sticky skeletonizers (Herb10), and Surface abrasion feeders (Herb11).

As regards plant species, pollinator frequencies and herbivory rates were registered for several species. The following species were considered: Azara microphylla (Flacourtiacea), Berberis darwinii (Berberidaceae), Embothrium coccineum (Proteaceae), Gaultheria mucronata (Ericaceae), Lomatia hirsuta (Proteaceae), Maytenus chubutensis (Celastraceae), Maytenus boaria (Celastraceae), Nothofagus antarctica (Nothofagaceae), Ribes cucullatum (Grossulariaceae), Ribes magellanicum (Grossulariaceae), Schinus patagonicus (Anacardiaceas). All of which are native forest species.

Within our network, more than two trophic levels were represented: a layer with pollinators and plant species, and another one with plant species and herbivore guilds. There are eight plants in common in both bipartite networks and in addition three plants exclusively have interaction only with herbivores. An adjacency matrix which was the result of projecting a network (Boccaletti et al., 2014) was used in this study. It contains 11 most representative woody plant species of a typical mixed woodland of northern Patagonia, 11 herbivore guilds and 25 pollinators grouped by morphotypes (Coulin et al., 2019). The abundance of each plant was quantified as cover percentage (Goldenberg et al., 2020).

\section{Results}

The previously described algorithm helped assess the significance of the topological structure of a network by adding a tunable degree of disorder while preserving the degree of each node. Subsequently, a step-by-step protocol was applied to identify the keystone species in our case study.

\subsection{Implementation of the algorithm}

Modularity was calculated as a function of the degree of randomness incorporated to the original network by flipping the adjacent nodes of two randomly chosen links. For each number of flips, we generated ten different networks and calculated the mean modularity as a function of that value. As shown below, modularity decreases as the number of changes increases (Fig. 2). Furthermore, the disorder of the network grew as the modularity values moved from the original ones to the corresponding random ones. This monotonous change is an indicator that the original network had some coded information that was destroyed when randomness was incorporated. Additionally, a hypothesis test was performed, where the t-statistic was used. A $p$-value of 0.031 was obtained and at the 0.05 level the null hypothesis was rejected. The conclusion was that after making a number of changes the obtained modularity is significantly different from the original one. 


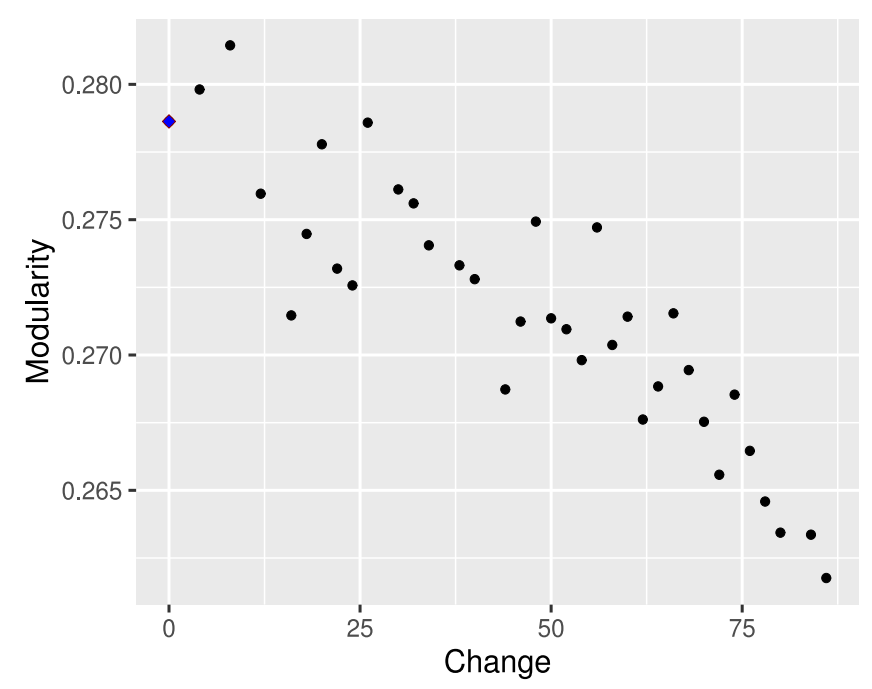

Fig. 2. Modularity vs. the number of changes (swappings) in the original network. Each value is a 10 network average except for the original one.

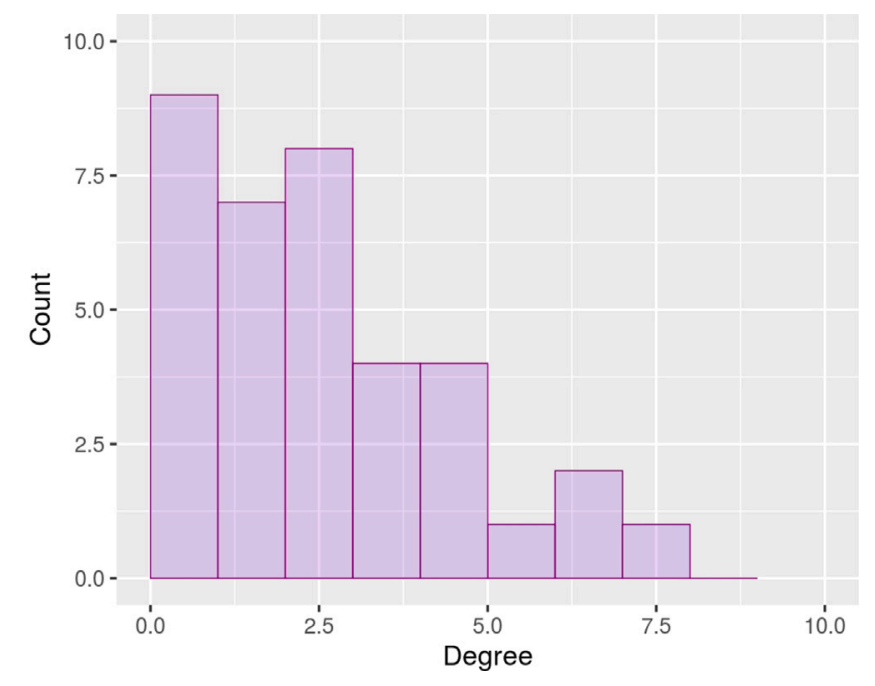

Fig. 3. Degree distribution of the original network nodes.

\subsection{Characterising our sampling}

In our sampling, the degree of each node was calculated, as well as the distribution of the resulting network. As shown in Fig. 3, node frequency shows typical attributes of the majority of ecological networks. The interesting feature is that the frequency of each node decays with the degree (Barabási and Réka, 1999). Nevertheless, this is not an indicator that our network is different from a random one, as with so few nodes it is almost impossible to distinguish a power-law distribution from an exponential random network (Newman, 2010). However, the shape of our distribution helps us detect a possible bias in our sampling.

The value of modularity, number of communities, density and the degree of each node were calculated. This network has 47 nodes, 111 links and its density is $\frac{111}{2162}(\approx 0.051)$. The average path length is 2.585 and its modularity 0.278 . A total of four communities were detected: 0 , $1,2,3$ with $34.04 \%, 21.28 \%, 14.89 \%$, and $29.79 \%$ of nodes, respectively (Fig. 4).

To determine keystone species, we constructed a list of species and normalised their betweenness to the highest value. An $\alpha=0.20$ was chosen because this value covered the region of interest and part of the region that is not (Table 1 ). Once the node with the greatest betweenness was removed, (R.magellanicum), the number of communities was calculated. Subsequently, E. coccineum was eliminated without deleting R.magellanicum and the number of communities was computed. Finally, when R.magellanicum and E. coccineum were removed separately, the number of communities and connected components increased in both cases, thereby indicating that these, in fact, were keystone species (Table 1 ). Note that $R$. magellanicum removal results in a more disconnected network. The betweenness centrality was calculated for all the nodes in the dataset, however the ones with the highest betweenness were the plants. Nonetheless, our focus group was "plants", as it may be easier to reintroduce a species to restore the ecosystem, as incorporating a pollinator or an arthropod species may be more difficult (Stanturf et al., 2014; Gatica-Saavedra et al., 2017)

Furthermore, the degree of each node was calculated and the two species with the highest degree were: R.magellanicum and E.coccineum. Despite the fact that, as a general rule, there is no correlation between degree and betweenness; in this case, we found one (Fig. 5A) by using Spearman's correlation $\rho=0.98$. Finally, we studied the correlation between abundance of each plant and betweenness; however, none was found (Spearman $\rho$ factor $=0.49$, Fig. $5 \mathrm{~B}$ ). We observed that nodes with the highest betweenness were not those with the highest abundance.

Moreover, the bipartite networks of pollinator-plant, herbivoreplant were analysed and it was observed that the keystone species are different than when analysing the pollinator-plant-herbivore guild network (Table A.1, Table A.2). If we analyse the plant network, which was obtained by projecting the plants belonging to the pollinator-plant network, we can no longer be sure which are the keystone species because the original information has been lost (Table A.3). The same applies to the plant network, which was built using the herbivore-plant network (Table A.4).

\section{Discussion}

An improved method was presented to qualitatively evaluate the changes in community division of a given network when randomness was gradually incorporated, erasing any valuable information stored in it but at the same time preserving some features intact. This method was used to search for keystone species in an ecological system. Consequently, we assessed the changes on the original network when an increasing amount of randomness was injected while preserving the degree distribution. It is noteworthy that the modularity decreased monotonically until a value corresponding to a completely random network was reached, with the constraint to preserve the original degree distribution. Finally, the node degree distribution was characterised and the keystone species were identified. Our results indicated that the structure of the network stored relevant information about the ecological system that in this case helped us detect the keystone species R.magellanicum and E.coccineum. Our work is different from previous studies which analysed bipartite network structures, centrality measures and species importance, fundamentally because we assessed, in advance, whether a network contained relevant information before working on it (Bascompte and Jordano, 2014; Burgos et al., 2007; Martín González et al., 2010; Medan et al., 2007; Memmott et al., 2004; Poisot et al., 2014; Vázquez and Simberloff, 2002). Moreover, we developed a method for validating the obtained results.

\subsection{Applications of the algorithm}

This original algorithm constitutes a valuable tool in order to decide whether a network contains relevant information in its system revealed through its community structure. In this work, as changes increased, the modularity value followed a decreasing tendency, indicating a loss of information in the system. Furthermore, with the increase in 


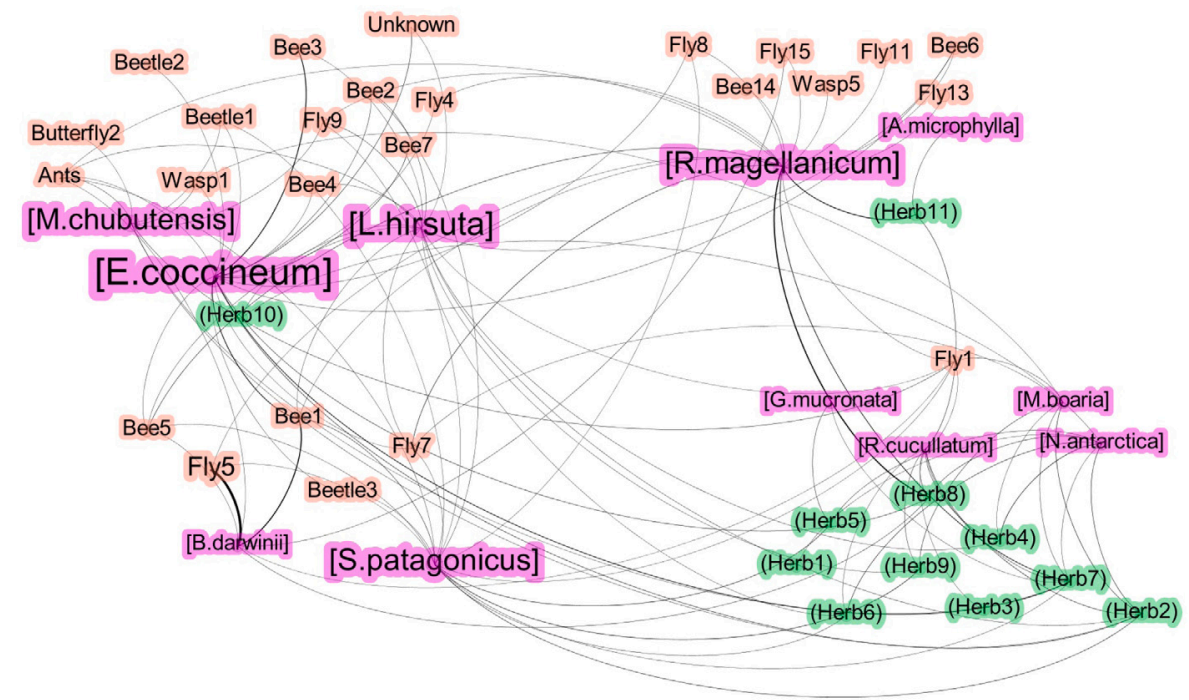

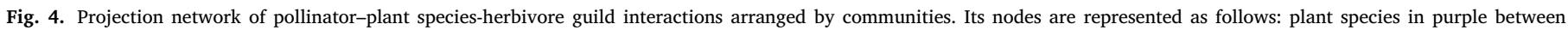
brackets, herbivore guilds in green between parenthesis and pollinators in orange.

Table 1

Changes in network structure after deleting a plant species. Only plant species with a normalised betweenness greater than $\alpha=0.20$ were considered.

\begin{tabular}{lllllll}
\hline $\begin{array}{l}\text { Deleted plant } \\
\text { species }\end{array}$ & Betweenness & $\begin{array}{l}\text { Normalised } \\
\text { betweenness }\end{array}$ & $\begin{array}{l}\text { Communities } \\
\text { (Before) }\end{array}$ & $\begin{array}{l}\text { Communities } \\
\text { (After) }\end{array}$ & $\begin{array}{l}\text { Connected } \\
\text { components } \\
\text { (Before) }\end{array}$ & $\begin{array}{l}\text { Connected } \\
\text { components } \\
\text { (After) }\end{array}$ \\
\hline R.magellanicum & 307.79 & 1 & 4 & 8 & 1 & 5 \\
E.coccineum & 256.16 & 0.83 & 4 & 6 & 1 & 3 \\
L.hirsuta & 151.88 & 0.49 & 4 & 5 & 1 & 1 \\
S.patagonicus & 145.82 & 0.47 & 4 & 4 & 1 & 1 \\
R.cucculatum & 93.36 & 0.30 & 4 & 5 & 1 & 2 \\
N.antarctica & 76.88 & 0.25 & 4 & 5 & 1 & 2 \\
M.chubutensis & 66.14 & 0.21 & 4 & & & 1 \\
\hline
\end{tabular}

A

B
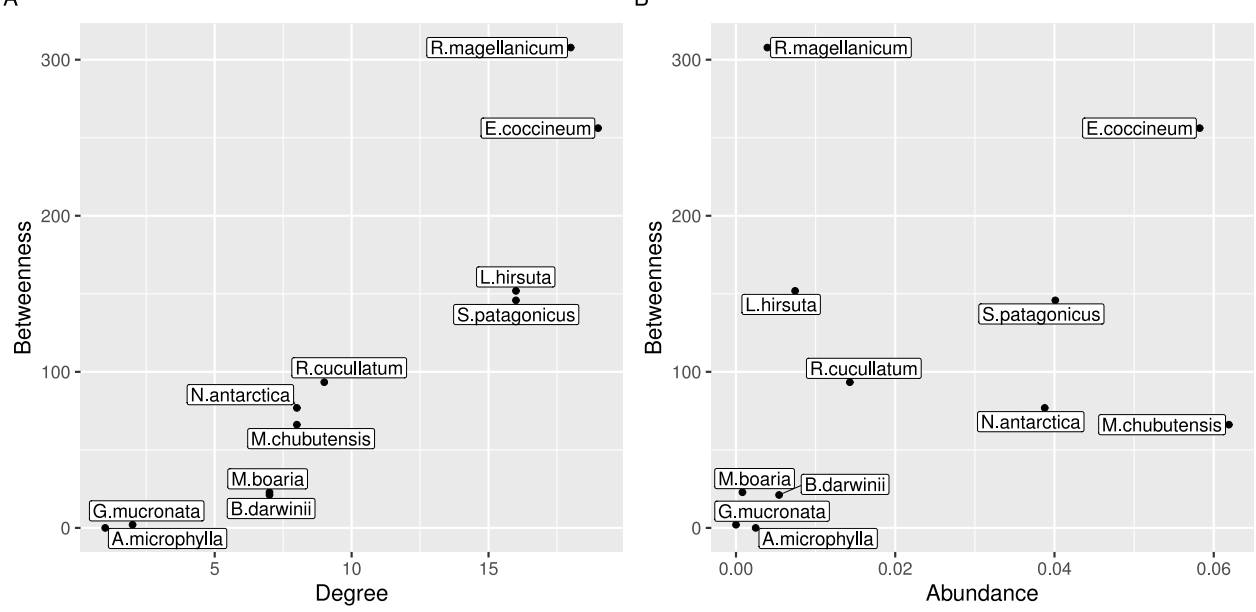

Fig. 5. (A) degree vs. betweenness and (B) abundance (Cover percentage) vs. betweenness of the 11 plant species.

disorder, the system lost its original structure and resembled a random network lacking community structure. Therefore, we can conclude that the studied network contained valuable information which could be quantified with further research. The random network constitutes our null model. If we do not have a suitable null model, we should change the methodology. The aforementioned algorithm can be applied in networks with more than two trophic levels; however, a projection ought to be made beforehand.

\subsection{Species identification}

The analysis of networks with more than two trophic levels constitutes a more complete approach than others previously developed with either herbivore-plant or pollinator-plant interactions. According to the data collected, the effect that each level has on the other can be taken into account by analysing the interactions jointly. Due to these species characteristics and results from prior studies, it was expected 
that N. antarctica, M. boaria and R. cucullatum would have stronger links with herbivore guilds than with pollinators (Nacif et al., 2021). In addition, $E$. coccineum and $R$. magellanicum have very attractive flowers and, at the same time, their leaves are highly palatable. These species can have high herbivory and pollinator interaction. Finally, E. coccineum was highly abundant among the sampled woody plant species.

In this analysis, the keystone species R.magellanicum and E.coccineum play a stabilising role in the ecosystem. Among woody forest species, these two, have relevant functions as regards ecological interactions but also as forest goods. Both species' flowers are highly preferred by pollinators such as insects and birds (Coulin et al., 2019) and its leaves support a high herbivore guild diversity (Nacif et al., 2021). E. coccineum, has high potential timber value for firewood, posts, and wood for furniture and, interestingly, its highly resprouting rate allows for alternative harvest management strategies (Donoso Zegers, 2013). Also, both species' leaves, bark, stem, and roots have several medical applications, Rapoport et al. (1999), Itkin (2004), and are appreciated as ornamental species, mainly E. coccineum (Gut, 2008). Moreover, the fruit of $R$. magellanicum, provides a valuable double service as food for forest animals and as a potential product for human consumption specifically as fresh berries, jam, preserves and liqueur (Hansen et al., 2013). Therefore, E.coccineum and R.magellanicum constitute keystone species through their relationship among pollinators, herbivores and woody plants of anthropic use, highlighting that monitoring these species and knowing their role in the ecological networks, constitute a priority for the conservation and management of these communities.

Furthermore, the construction of network communities could show interesting outcomes regarding its composition. In our case, we found interesting differences among the four identified communities, varying in terms of pollinators, plant species, and guild herbivore composition. Particularly, those communities whose plants were highly visited by pollinators were less preferred by herbivores. Plant species with typical attractive flowers for insect pollinators belong to three of the above mentioned communities (Raffaele et al., 2014). It is worth noting that these plants also have less palatable leaves, except for R.magellanicum (Fajardo and Siefert, 2016). On the other hand, the community which was consumed at the highest rate by different herbivore guilds was composed of plants which were less frequently pollinated by insects. G.mucronata, M.boaria, N.antarctica, and R.cucullatum have high quality leaves ( Blackhall et al., 2012; Nacif et al., 2021). Hence, this mathematical approach could become a useful strategy to determine whether an equilibrium exists in the network layers providing information for further analysis. Overall, the obtained results make sense in biological terms and invite us to replicate the approach in other study systems or by extending this one.

Another method for identifying keystone species is to determine the abundance of each plant species, yet it depends on the issue in question. In our case, a non-correlation between abundance and betweenness was found. Additionally, the most abundant species was M.chubutensis. Although, the pollinators have access to its flowers, it is less preferred by pollinators and removing it does not generate changes in the structure of the network. Interestingly, E.coccineum is not only our second keystone species, but it is also the second most abundant one having the highest degree among the 11 plant species. In fact, when keystone species are considered by degree, these are E.coccineum and R.magellanicum. This is, most likely, a consequence of a positive correlation between betweenness and degree which are generally not correlated.

In recent years, conservation biology and management have focused on the study of keystone species, whose definition and quantification remains challenging. Jordán and Scheuring (2002), who studied and compared 13 centrality indices in ecological networks, determined that when links are not weighted, betweenness is the best index for identifying species' positional importance. Moreover, keystone species identified in networks of more than two trophic levels can be different from those found in bipartite ones with two trophic levels. In our case, we identified keystone species in bipartite mutualistic and antagonistic networks by using the method presented above. In the former one, the keystone species were R.magellanicum and E.coccineum (Table A.1, Fig. A.1), and in the latter one, R.cucculatum and N.antarctica (Table A.2, Fig. A.2). This shows that keystone species in the whole system are not necessarily the same as in bipartite networks. Clearly, we might lose important details of the ecosystem when only two trophic levels are considered in the proposed network.

While in this study, we only considered pollinator-plant-herbivore guild networks, our method can be applied to other types of networks. In either of these ecological network types, there are ample opportunities for further analyses, where it might render itself useful, for example, to identify species' common features belonging to the same community. Furthermore, as a mathematical approach, our algorithm could be applied to a wide variety of disciplines where the network keystone nodes would allow us to determine which elements play a central role. In this way, researchers should verify whether their network contains valuable information. We hope that future studies will apply the proposed method in order to carry out a thorough network analysis before drawing anticipatory conclusions.

\section{CRediT authorship contribution statement}

Claudia A. Huaylla: Conceptualization, Methodology, Software, Formal analysis, Writing - original draft, Writing - review \& editing. Marcos E. Nacif: Data curation, Writing - original draft, Writing review \& editing. Carolina Coulin: Data Curation, Writing - original draft, Writing - review \& editing. Marcelo N. Kuperman: Conceptualization, Methodology, Formal analysis, Writing - original draft, Writing - review \& editing, Supervision. Lucas A. Garibaldi: Conceptualization, Methodology, Formal analysis, Data curation, Writing - original draft, Writing - review \& editing.

\section{Declaration of competing interest}

The authors declare that they have no known competing financial interests or personal relationships that could have appeared to influence the work reported in this paper.

\section{Data available}

The code for these analyses is available in GitHub https://github.com/claudiah85/Huaylla.et.al.

\section{Acknowledgements}

We would like to thank Néstor Pérez Mendéz, Juan Ignacio Agüero and Damasia Lozada for comments and suggestions on the manuscript. We appreciate funding from Agencia Nacional de Promoción Científica y Tecnológica, Argentina (PICT 2016-0305), Universidad Nacional de Río Negro, Argentina (PI 40-13-635), BiodivERsA-Belmont Forum (OBSERV), Argentina, Ministerio de Ciencia y Tecnología, Argentina (GILES - ERANet-LAC2015/T010880).

\section{Appendix A. Supplementary data}

Supplementary material related to this article can be found online at https://doi.org/10.1016/j.ecolmodel.2021.109734. 


\section{References}

Barabási, A.L., Réka, A., 1999. Emergence of scaling in random networks. Science 286 (5439), 509-512. http://dx.doi.org/10.1126/science.286.5439.509.

Bascompte, J., 2007. Networks in ecology. Basic Appl. Ecol. 8 (6), 485-490. http: //dx.doi.org/10.1016/j.baae.2007.06.003.

Bascompte, J., Jordano, P., 2014. Mutualistic Networks. Princeton University Press.

Bascompte, J., Jordano, P., Melian, C.J., Olesen, J.M., 2003. The nested assembly of plant-animal mutualistic networks. Proc. Natl. Acad. Sci. USA 100 (16), 9383-9387. http://dx.doi.org/10.1073/pnas.1633576100.

Blackhall, M., Raffaele, E., Veblen, T.T., 2012. Is foliar flammability of woody species related to time since fire and herbivory in northwest Patagonia, Argentina? J. Veg. Sci. 23 (5), 931-941. http://dx.doi.org/10.1111/j.1654-1103.2012.01405.x.

Blondel, V.D., Guillaume, J.-L., Lambiotte, R., Lefebvre, E., 2008. Fast unfolding of communities in large networks. J. Stat. Mech. Theory Exp. (10), http://dx.doi.org/ 10.1088/1742-5468/2008/10/P10008.

Boccaletti, S., Bianconi, G., Criado, R., Genio, C.I., Gómez-Gardeñez, J., Romance, M., Sendiña Nadal, I., Wang, Z., Zanin, M., 2014. The structure and dynamics of multilayer networks. Phys. Rep. 544 (1), 1-122. http://dx.doi.org/10.1016/j. physrep.2014.07.001.

Burgos, E., Ceva, H., Perazzo, R.P., Devoto, M., Medan, D., Zimmermann, M., María Delbue, A., 2007. Why nestedness in mutualistic networks? J. Theoret. Biol. 249 (2), 307-313. http://dx.doi.org/10.1016/j.jtbi.2007.07.030.

Cottee-Jones, H.E.W., Whittaker, R.J., 2012. The keystone species concept: a critical appraisal. Front. Biogeogr. 4 (3), 117-127. http://dx.doi.org/10.21425/ F5FBG12533.

Coulin, C., Aizen, M., Garibaldi, L., 2019. Contrasting responses of plants and pollinators to wood-land disturbance. Wiley Online Libr. Austral Ecol. 44 (6), 1040-1051. http://dx.doi.org/10.1111/aec.12771.

Donoso Zegers, C., 2013. Las especies arbóreas de los bosques templados de Chile y Argentina: autoecología. Universidad Austral de Chile, Valdivia: Cuneo.

Dormann, Carsten, 2011. How to be a specialist? Quantifying specialisation in pollination networks. Netw. Biol. 1, 1-20.

Fajardo, A., Siefert, A., 2016. Phenological variation of leaf functional traits within species. Oecologia 180, 951-959. http://dx.doi.org/10.1007/s00442-016-3545-1.

Fornito, A., Bullmore, E.T., Zalesky, A., 2016. Fundamentals of Brain Network Analysis. Elsevier Science, San Diego, CA, USA.

Freeman, L.C., 1979. Centrality in social networks, conceptual clarification. Social Networks 1 (3), 215-239. http://dx.doi.org/10.1016/0378-8733(78)90021-7.

Garibaldi, L.A., Kitzberger, T., Chaneton, E.J., 2011. Environmental and genetic control of insect abundance and herbivory along a forest elevational gradient. Oecologia 167, 117-129. http://dx.doi.org/10.1007/s00442-011-1978-0.

Gatica-Saavedra, P., Echeverría, C., Nelson, C.R., 2017. Ecological indicators for assessing ecological success of forest restoration: A world review. Restoration Ecol. 25, 850-857. http://dx.doi.org/10.1111/rec.12586.

Girvan, M., Newman, M.E.J., 2002. Community structure in social and biological networks. Proc. Natl. Acad. Sci. 99 (12), 7821-7826. http://dx.doi.org/10.1073/ pnas.122653799.

Goldenberg, M.G., Oddi, F.J., Gowda, J.H., Garibaldi, L.A., 2020. Effects of firewood harvesting intensity on biodiversity and ecosystem services in shrublands of northern patagonia. Forest Ecosyst. 7 (47), http://dx.doi.org/10.1186/s40663-02000255-y.

Gut, B., 2008. Trees in Patagonia. Birkhäuser, Switzerland.

Hansen, N., Codesal, P., Quinteros, P., Gallardo, C., 2013. Especies del sotobosque en ambientes de Nire en Chubut. INTA, Buenos Aires, E.E.A:Esquel.

Itkin, S., 2004. Plantas de la Patagonia Para la Salud. Editorial Caleuche, Bariloche.

Jeong, H., Mason, S.P., Barabási, A.L., Oltvai, Z.N., 2001. Lethality and centrality in protein networks. Nature 411 (6833), 41-42. http://dx.doi.org/10.1038/35075138.

Jeong, H., Tombor, B., Albert, R., Oltvai, Z.N., Barabási, A.-L., 2000. The large-scale organization of metabolic networks. Nature 407, 651-654. http://dx.doi.org/10. 1038/35036627.
Jordán, Ferenc, 2009. Keystone species and food webs. Philos. Trans. R. Soc. London [Biol.] 364, 1733-1741. http://dx.doi.org/10.1098/rstb.2008.0335.

Jordán, F., Liu, W.-C., Davis, A.J., 2006. Topological keystone species: measures of positional importance in food webs. Oikos 112 (3), 535-546. http://dx.doi.org/10, 1111/j.0030-1299.2006.13724.x.

Jordán, F., Scheuring, I., 2002. Searching for keystones in ecological networks. Oikos 99, 607-612. http://dx.doi.org/10.1034/j.1600-0706.2002.11889.x.

Jordán, F., Takács-Sánta, A., Molnár, I., 1999. A reliability theoretical quest for keystones. Oikos 86 (3), 453-462. http://dx.doi.org/10.2307/3546650.

Kivelä, M., Arenas, A., Barthelemy, M., Gleeson, J., Porter, M., 2014. Multilayer networks. J. Complex Netw. 2 (3), 203-271. http://dx.doi.org/10.1093/comnet/ cnu016.

Martín González, A.M., Dalsgaard, B., Olesen, J., 2010. Centrality measures and the importance of generalist species in pollination networks. Ecol. Complex. 7 (1), 36-43. http://dx.doi.org/10.1016/j.ecocom.2009.03.008.

McQuillan, P.B., 2008. Nothofagus (Fagaceae) and its invertebrate fauna-an overview and preliminary synthesis. Biol. J. Linnean Soc. 49 (4), 317-354. http://dx.doi.org/ 10.1111/j.1095-8312.1993.tb00910.x.

Medan, D., Perazzo, R.P.J., Devoto, M., Burgos, E., Zimmermann, M.G., Ceva, H., Delbue, A.M., 2007. Analysis and assembling of network structure in mutualistic systems. J. Theor. Biol. 246, 510-521. http://dx.doi.org/10.1016/j.jtbi.2006.12. 033.

Mello, M.A.R., Rodrigues, F.A., Costa, L.F., Kissling, W.D., Sekercioglu, C.H., Maquitti, F.M.D., Kalko, E.K.V., 2015. Keystone species in seed dispersal networks are mainly determined by dietary specialization. Oikos 124, 1031-1039. http: //dx.doi.org/10.1111/oik.01613.

Memmott, J., Waser, N.M., Price, M.V., 2004. Tolerance of pollination networks to species extinctions. Proc. R. Soc. B Biol. Sci. 271 (1557), 2605-2611. http://dx. doi.org/10.1098/rspb.2004.2909.

Nacif, M., Kitzberger, T., Garibaldi, L., 2020. Positive outcomes between herbivore diversity and tree survival: Responses to management intensity in a Patagonian forest. Forest Ecol. Manag. 458. http://dx.doi.org/10.1016/j.foreco.2019.117738.

Nacif, M., Quintero, C., Garibaldi, L., 2021. One-third harvesting intensity enhances native tree performance of contrasting species while conserving herbivore diversity in a Patagonian woodland. p. 483. http://dx.doi.org/10.1016/j.foreco.2020.118719.

Newman, M.E.J., 2004a. Analysis of weighted networks. Phys. Rev. E 70, 056131-056140. http://dx.doi.org/10.1103/PhysRevE.70.056131.

Newman, M.E.J., 2004b. Fast algorithm for detecting community structure in networks. Phys. Rev. E 69 (6), 066133. http://dx.doi.org/10.1103/PhysRevE.69.066133.

Newman, MJournal of Animal Ecology.E.J., 2010. Networks: An Introduction. Oxford University Press, Oxford.

Novotny, V., Miller, S., Baje, Leontine, Balagawi, S., Basset, Y., Cizek, L., Craft, K.J., Dem, F., Drew, R.A.I., Hulcr, J., Leps, Jan, Lewis, O.T., Pokon, R., Stewart, A.J.A., Allan Samuelson, G., Weiblen, G.D., 2010. Guild-specific patterns of species richness and host specialization in plant-herbivore food webs from a tropical forest. J. Anim. Ecol. 79 (6), 1193-1203. http://dx.doi.org/10.1111/j.1365-2656.2010.01728.x.

Poisot, T., Stouffer, D., Gravel, D., 2014. Beyond species: Why ecological interaction networks vary through space and time. Oikos 124 (3), 243-251. http://dx.doi.org/ 10.1111/oik.01719.

Raffaele, E., De Torrres Curth, M., Morales, C.L., Kitzberger, T., 2014. Ecología e historia natural de la Patagonia Andina. Un cuarto de siglo de investigación en biogeografía, ecología y conservación Editorial. Fundación de Historia Natural Félix de Azara, Buenos Aires, p. 256.

Rapoport, E., Ladio, A., Sanz, E., 1999. Plantas Nativas Comestibles de la Patagonia Andina, Argentino-Chilena. Parte I. Universidad Nacional del Comahue y National Geographic Society, p. 81.

Ravasz, E., Somera, A.L., Mongru, D.A., Oltvai, Z.N., Barabási, A.L., 2002. Hierarchical organization of modularity in metabolic networks. Science 297 (5586), 1551-1555. http://dx.doi.org/10.1126/science.1073374.

Stanturf, J.A., Palik, B.J., Kasten Dumroese, R., 2014. Contemporary forest restoration: A review emphasizing function. Forest Ecol. Manag. 331, 292-323. http://dx.doi. org/10.1016/j.foreco.2014.07.029.

Vázquez, D.P., Simberloff, D., 2002. Ecological specialization and susceptibility to disturbance: conjectures and refutations. Amer. Nat. 159 (6), 606-623. http://dx. doi.org/10.1086/339991. 